\title{
Distribution of personality: Types or skewness?
}

\author{
Kentaro Katahira*,1, Yoshihiko Kunisato ${ }^{2}$, Yuichi Yamashita ${ }^{3}$, and Shinsuke Suzuki ${ }^{*, 4}$ \\ ${ }^{1}$ Department of Psychological and Cognitive Sciences, Graduate School of Informatics, Nagoya \\ University, Japan. \\ ${ }^{2}$ Department of Psychology, Senshu University, Japan, \\ ${ }^{3}$ Department of Functional Brain Research, National Institute of Neuroscience, National Center \\ of Neurology and Psychiatry, Japan. \\ ${ }^{4}$ Brain, Mind and Markets Laboratory, Department of Finance, The University of Melbourne, \\ Australia.
}

*Correspondence should be addressed to Kentaro Katahira (katahira.kentaro@b.mbox.nagoyau.ac.jp) and Shinsuke Suzuki (shinsuke.szk@gmail.com).

What kinds of personalities do humans have? Can these personalities be classified into several discrete types? These issues have been of considerable concern as they could potentially provide deeper understanding of the nature of human individuality and mental disorders. Recently, Gerlach et al. ${ }^{1}$ addressed these issues by applying established machine-learning techniques to big data (more than 1.5 million respondents in total). They found four meaningful clusters in personality dimensions, suggesting the existence of at least four personality types. Here, we propose an alternative interpretation of their result: a skewed shape of the distribution with no cluster structures in personality space can erroneously lead to the seemingly meaningful clusters reported in Gerlach et al. Our finding reopens the question regarding the existence of personality types.

It is now widely accepted that human personality is characterized by five dimensions (traits or factors), which consist of neuroticism, extraversion, openness, agreeableness, and conscientiousness $^{2}$. Yet, understanding of how human personalities are distributed in this five-dimensional (5D) space remains elusive. There exist at least two major views: the dimensional view and categorical view. The dimensional view supposes that the distribution is unimodal and individuals' personalities are continuously distributed in the 5D space. The categorical view posits that there are multiple clusters (dense regions) in personality space (i.e. the distribution is multimodal) and each individual can be classified into one of these clusters. In personality theory, such clusters are referred to as personality 'types'. While common analytical tools of personality (e.g. factor analysis ${ }^{3}$ ) implicitly assume the dimensional view, some researchers have taken the categorical view and claimed the existence 
of personality types (e.g. Robins et al. ${ }^{4}$ ).

A recent study by Gerlach et al. ${ }^{1}$ aimed to identify personality types in a highly robust manner, by analysing four large data sets. Their analyses, which were based on machine learning and statistical techniques, identified four meaningful clusters deemed as personality types, supporting the categorical view. However, in the present study, we provide evidence for the possibility that Gerlach et al.'s analysis cannot exclude the dimensional view. Specifically, we performed computer simulations to show that a skewed distribution without cluster structure can lead to spurious clusters that are deemed 'meaningful clusters' or 'types' by Gerlach et al.'s analysis.

The core part of Gerlach et al.'s ${ }^{1}$ analysis is fitting Gaussian mixture models (GMM) to the five factor scores that provide the positions of individuals in the 5D space (the procedure adopted in Gerlach et al. ${ }^{1}$ is briefly described in Supplementary Text 1). GMM represents a given distribution by weighted sum of a finite number of Gaussian (normal) distributions. If there are indeed cluster structures and each cluster can be represented by single Gaussian distribution, each Gaussian component may correspond to a single cluster (Fig. 1a, b). To examine whether each Gaussian component is a truly meaningful cluster, they performed a statistical test based on the null model that assumes the five factors are distributed independently of each other. As a result, they identified four Gaussian components as meaningful clusters.

However, even if the target distribution is unimodal and there is no cluster structure, similar results (i.e. emergence of meaningful clusters) can be obtained when the distribution has skewness and deviates from a pure Gaussian distribution (Fig. 1c, d). This phenomenon occurs because the GMM represent a non-Gaussian distribution by combining multiple Gaussian components $\left(\right.$ refs $\left.^{5,6}\right)$. The samples in Fig. 1c were artificially generated from a unimodal, skewed distribution ${ }^{7}$ (see Supplementary Text 2). For this case, the best fitted GMM has seven components to represent the skewed distribution (Fig. 1d). Among seven components, three are deemed 'meaningful clusters' according to the statistical criteria of Gerlach et al. (Supplementary Fig. 1). Because the true distribution is unimodal and has no cluster structure by construction, this result is due to model misspecification.

This could have happened in Gerlach et al.'s analysis ${ }^{1}$. According to Gerlach et al., the centre of each cluster should have higher density compared to other regions. In contrast to this prediction, the density surface on a $2 \mathrm{D}$ plane spanned by three meaningful clusters (Supplementary Fig. 5 of ref ${ }^{1}$ ) implies that the cluster centres do not have clear density peaks. Additionally, the marginal 2D distributions of factor scores (Supplementary Fig. 2 of ref ${ }^{1}$ ) appear to be skewed. Although the skewness of these distribution seems to be smaller compared to the distribution in our simulation of a $2 \mathrm{D}$ case (Fig. 1c), the effect of skewness 
might be more manifested in a 5D space. From the above, we suggest the possibility that the results of Gerlach et al. ${ }^{1}$ do not necessarily reflect the cluster structures, and instead could reflect skewness of the personality distribution.

The skewness of the distribution as well provides information for understanding the mechanisms of how individual differences in personality arise. For example, the skewness embedded in multiple factors may be caused by higher-order factors of personality ${ }^{8}$, which may obey a skewed distribution, although there is another possibility that the skewness is caused by mere range restriction of responses (see $\mathrm{ref}^{9}$ ).

To conclude, in the present study, we have demonstrated the possibility that the skewness of the distribution can influence the personality types reported by Gerlach et al. ${ }^{1}$, although we did not formally evaluate how much their results indeed suffered from this skewness. A formal evaluation requires novel statistical methods that can represent and quantify the skewness of a multivariate distribution appropriately. Our demonstration suggests that, despite the seminal work by Gerlach, it is still an open question whether the distribution of personality should be characterized as categorical, dimensional, or intermediate between them. Given that personality has been of great interest to people, investigating these issues would contribute to the progress of not only personality psychology, but also statistical science and machine learning.

Acknowledgements

This work was supported by JSPS KAKENHI Grant Numbers 17H05946 and 19H04902 (K.K.), 16H05957 (Y.K.), JP17H06039 (Y.Y.) and 17H05933 (S.S.), and JST CREST Grant Number JPMJCR16E2 (Y.Y.). The authors thank Asako Toyama for reading and checking the manuscript.

\section{References}

1. Gerlach, M., Farb, B., Revelle, W. \& Amaral, L. A. N. A robust data-driven approach identifies four personality types across four large data sets. Nat. Hum. Behav. 2, 735-742 (2018).

2. Goldberg, L. R. An alternative" description of personality": the big-five factor structure. J. Pers. Soc. Psychol. 59, 1216 (1990).

3. Cattell, R. The scientific use of factor analysis in behavioral and life sciences. (Springer Science \& Business Media, 2012).

4. Robins, R. W., John, O. P., Caspi, A., Moffitt, T. E. \& Stouthamer-Loeber, M. Resilient, overcontrolled, and undercontrolled boys: three replicable personality types. J. Pers. Soc.

Psychol. 70, 157 (1996). 
5. Roeder, K. \& Wasserman, L. Practical Bayesian density estimation using mixtures of normals. J. Am. Stat. Assoc. 92, 894-902 (1997).

6. Bauer, D. J. \& Curran, P. J. The Integration of Continuous and Discrete Latent Variable Models: Potential Problems and Promising Opportunities. Psychol. Methods 9, 3-29 (2004).

7. Azzalini, A. The skew-normal distribution and related multivariate families. Scand.J. Stat. 32, 159-188 (2005).

8. Deyoung, C. G. Higher-order factors of the big five in a multi-informant sample. J. Pers. Soc. Psychol. 91, 1138-1151 (2006).

9. Rice, K. G. \& Richardson, C. M. E. Classification challenges in perfectionism. J. Couns. Psychol. 61, 641-648 (2014). 
Figure

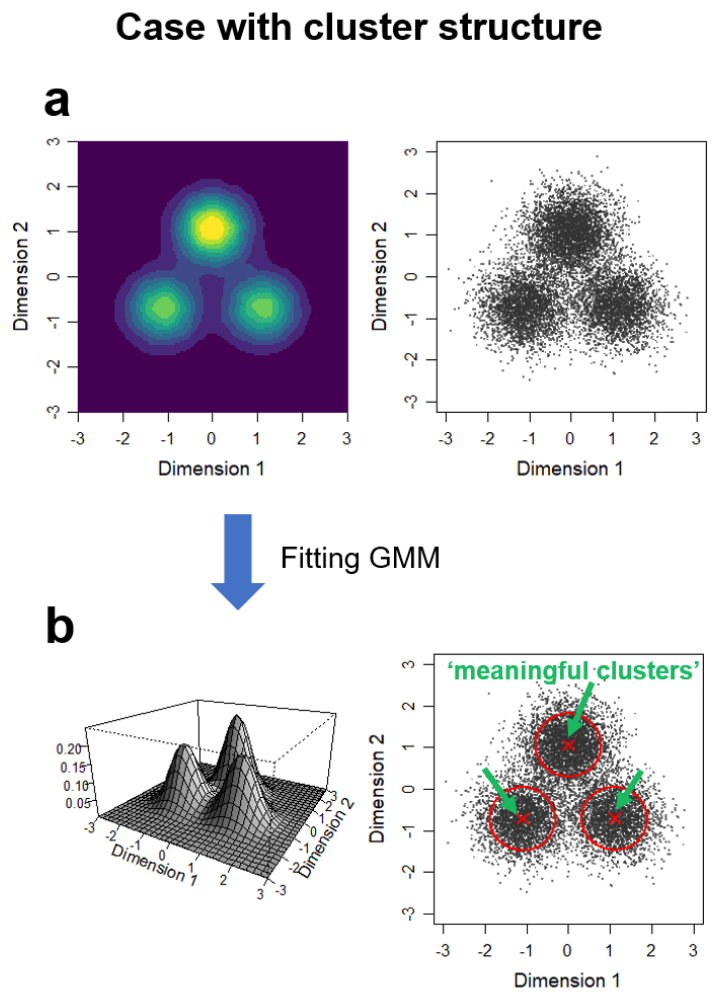

Case with distribution skewness (no cluster structure)

C
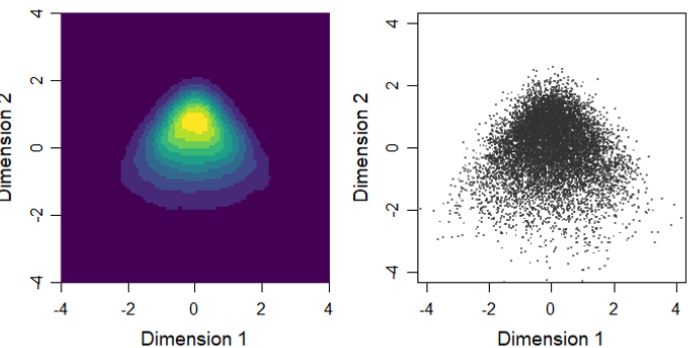

Fitting GMM

d

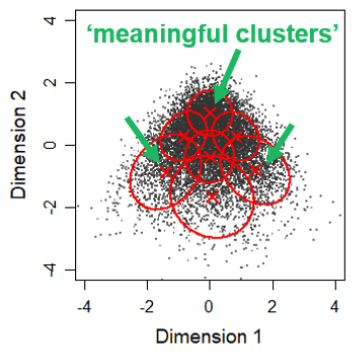

Figure 1: Illustration of how clustering based on a Gaussian mixture model works for two types of distribution

Comparison of a case with a clear cluster structure $(\mathbf{a} \& \mathbf{b})$ and a case with a skewed distribution (c\&d). a \& c, The right panel for each shows the scatter plot of synthesized data and the left shows the estimated probability density from the samples. b \& d, Result of fitting Gaussian mixture models. The left panel shows the probability density functions of the fitted GMM. The right panel indicates the one standard deviation contours and the centres of the estimated Gaussian components (the scatter plots which are identical with the above panels are shown). The Gaussian components deemed 'meaningful clusters' by Gerlach et al.'s procedure are indicated by green arrows (see Supplementary Text 2 and Supplementary Fig. 1 for details). 


\section{Supplementary Information}

\section{Supplementary Text 1: Procedures of analysis in Gerlach et al.}

Here, we briefly summarize the analysis procedure adopted in Gerlach et al. ${ }^{1}$ First, from responses to the questionnaires, five latent dimensions which correspond to the five main domains of the Big Five model are extracted by exploratory factor analysis ${ }^{2}$, assuming the orthogonality of the factors. Then, the five-factor scores for each respondent are estimated. By these scores, each respondent is localized in a 5-dimensional (D) space of personality.

Next, to find clusters in the distribution of factor scores, Gaussian mixture models (GMM) are fitted to the estimated five-factor scores. GMM represents a given distribution by a weighted average of a finite number of Gaussian (normal) distributions. Each Gaussian component is a candidate of a single cluster. The number of Gaussian components is determined from data by using the Bayesian information criterion (BIC), a common measure for statistical model selection. The smaller BIC indicates a more probable model.

Then, the Gaussian components are checked as to whether they are truly meaningful clusters. To achieve this, the probability densities at the centre of each component in 5D space are estimated by using the kernel density estimation. The estimates are compared to 'a null model' that corresponds to the density function averaged over estimated densities from 1,000 randomised (shuffled) data sets, which destroys the relationship between the five factors. The clusters whose centres have a significantly higher density $(p<0.01$, and the density ratio, called the 'enrichment' is more than 1.25) compared to the null model are deemed as meaningful clusters.

\section{Supplementary Text 2: Simulation procedure}

By using the synthesized data set, we illustrate how Gerlach et al.'s analysis works for two scenarios (Fig. 1 of the main text). Here, we concern 2D space, rather than 5D space as in Gerlach et al., for illustrative purposes. For the case with cluster structures, we generated samples from GMM with three components, whose means are (dimension 1, dimension 2$)=$ $(0,2),(-1.5,-0.5)$ and $(1.5,-0.5)$ and all the variance-covariance matrices are diagonal matrices whose diagonal components are 0.5 . The mixing proportions are $0.4,0.3$, and 0.3 , respectively. The resulting samples are standardized so that each variable has zero mean and unit variance.

For the case with a skewed distribution, we first generated samples from two independent skew-normal distributions ${ }^{5}$ with parameters $\left(\operatorname{ref}^{3}\right)$ : $\xi=-1, \omega=1, \alpha=4$ (for the first 
variable) / -4 (for the second variable; Supplementary Fig. 1a). Then the samples were rotated by 45 degrees (Supplementary Fig. 1b). This rotation is crucial for observing spurious clusters that are deemed meaningful, as mentioned later. By construction, this data has no (multiple) cluster structure.

We fitted GMMs to the generated samples (Fig. 1b, d of the main text). For the case with a cluster structure, we found that the model with three Gaussian components is appropriate (it gave the minimum BIC). For the case with a skewed distribution, we found that the model with seven Gaussian components is appropriate.

Next, we examined whether each Gaussian component obtained by GMM is a meaningful cluster. We constructed a null model by re-assigning the factors across samples (Supplementary Fig. 1c; here, only the case with a skewed distribution is shown). We note that the density surface of the null model has a different shape to that of the original data (Supplementary Fig. 1b). This is because the skewness of the synthesized data was embedded in the joint distribution of two variables by rotation: the shuffling procedure destroyed this skewness. As a result, there were regions where the density of the original data was significantly higher than the null model, which gave higher values of enrichment and smaller $p$-values (Supplementary Fig. 1e). In this case, the centre of the three components fell into these regions. These three components are identified as 'meaningful clusters'.

It should be noted that what influences the emergence of spurious meaningful clusters is not the skewness of marginals (distribution of each variable where the other variable is marginalized out), rather, it is the skewness embedded in a joint distribution of more than one factor. Although the skewness of marginals can lead to multiple Gaussian components being used, it does not solely produce meaningful clusters, because the null model has the same marginals as the original distribution (compare Supplementary Fig. $1 \mathrm{~b}$ and Supplementary Fig.1c). That is why we rotated the samples in the simulation of the skewed distribution case to demonstrate this effect. 
Figure

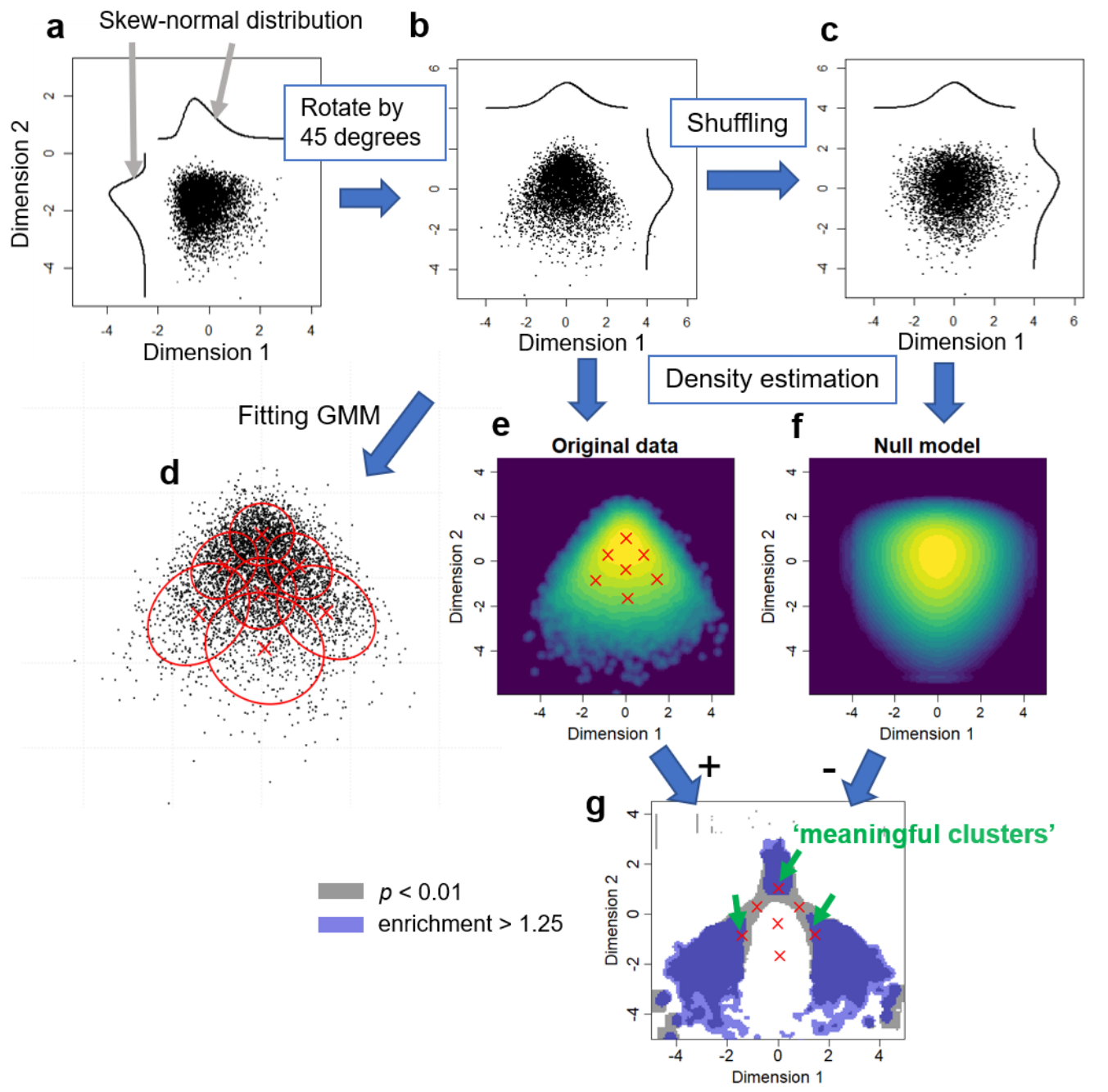

Supplementary Figure 1: Illustration of how a spurious 'meaningful cluster' emerges from a skewed distribution.

a, b. Synthesized data. The values of two variables are drawn from skew-normal distributions (a) and then rotated by 45 degrees (b). c. The random null model is obtained by shuffling the values of each dimension (destroying the relationship between dimensions). d. Gaussian mixture models. Red crosses indicate the centre (mean) of the Gaussian components. Red ellipses indicate the one standard deviation contours of the Gaussian components. e. Log probability density of the original (synthesized) data obtained by using kernel density estimation. f. Log probability density of a random null model. g. Evaluation of clusters. The Gaussian components whose means fall into grey-shaded regions $(p<0.01)$ and blue-shaded regions (enrichment $>1.25$ ) are deemed 'meaningful clusters'. 


\section{References}

1. Gerlach, M., Farb, B., Revelle, W. \& Amaral, L. A. N. A robust data-driven approach identifies four personality types across four large data sets. Nat. Hum. Behav. 2, 735-742 (2018).

2. Cattell, R. The scientific use of factor analysis in behavioral and life sciences. (Springer Science \& Business Media, 2012).

3. Azzalini, A. The skew-normal distribution and related multivariate families. Scand. J. Stat. 32, 159-188 (2005). 